\title{
ESTEGOSAURIOS (DINOSAURIA) DEL JURÁSICO SUPERIOR- CRETÁCICO INFERIOR DE LA COMARCA DE LOS SERRANOS (VALENCIA, ESPAÑA)
}

\author{
$M^{a}$ Lourdes CASANOVAS-CLADELLAS', Jose Vicente \\ SANTAFÉ-LLOPIS', Carles SANTISTEBAN BOVÉ' y Xavier \\ PEREDA SUBERBIOLA ${ }^{3}$ \\ 'Institut de Paleontologia "M. Crusafont". Escola Industrial 23. 08201 Sabadell. \\ ? Universidad de Valencia. Depart. de Geología. Dr. Moliner 50. 46100 Burjassot (Valencia). \\ "Universidad del País Vasco/Euskal Herriko Unibertsitatea. Fac. Ciencias. Depart. Paleontología. \\ Apartado 644. 48080 Bilbao.
}

\begin{abstract}
Casanovas-Cladellas, M.L., Santafé-Llopis, J.V., Santisteban Bové, C. y Pereda Suberbiola, X. 1999. Estegosaurios (Dinosauria) del Jurásico Superior-Cretácico Inferior de la Comarca de los Serranos (Valencia, España). [Upper Jurassic-Lower Cretaceous stegosaurian dinosaurs from Los Serranos area (Valencia, Spain).] Revista Española de Paleontología, nextr. Homenaje al Prof. J. Truyols, 57-63. ISSN 0213-6937.
\end{abstract}

\begin{abstract}
A synthesis is presented of all the stegosaurian remains from the Aras de Alpuente area (Losilla and Cerrito del Olmo localities, Valencia Province), including the description of a new femur. Moreover, the stratigraphic distribution of the stegosaurian and other dinosaurian localities from the same region is discussed. The stegosaurian material from Valencia was regarded as Lower Cretaceous (Wealden facies) in earlier papers. However, it is likely that the Losilla and Cerrito del Olmo localities are of Late Jurassic age (Purbeck facies). The presence of the stegosaurian Dacentrurus armatus (Owen) supports this assumption.
\end{abstract}

Keywords: Stegosaurs, Dinosaurs, Upper Jurassic, Lower Cretaceous, Los Serranos, Valencia.

\section{RESUMEN}

Se describe un fémur y se hace una síntesis del material de estegosaurios hallado hasta el momento en los alrededores de Aras de Alpuente (Valencia), en las localidades de Losilla y Cerrito del Olmo. Se indica la situación estratigráfica de las mencionadas localidades y se establece su relación con los restantes yacimientos de la zona que han proporcionado restos de dinosaurios. Aunque en las anteriores publicaciones se considera que el material es del Cretácico Inferior, no se descarta que Losilla y Cerrito del Olmo puedan ser consideradas como Jurásico terminal. Hasta ahora, sólo la presencia en ellas del estegosaurio Dacentrurus armatus (Owen) puede hacernos sospechar esta posibilidad.

Palabras clave: Estegosaurios, Dinosaurios, Jurásico Superior, Cretácico Inferior, Los Serranos, Valencia.

\section{INTRODUCCIÓN}

En 1995, dimos a conocer, por primera vez, la presencia de estegosaurios en España (Casanovas et al, 1995b). En aquel momento sólo se había hallado una espina caudal en la localidad de Losilla (Aras de Alpuente, Valencia). Dicha espina, fragmentaria, tiene una longitud de unos $55 \mathrm{~cm}$, faltando el extremo proximal y su parte medial. El escaso valor diagnóstico de la pieza hizo que debiera asignarse a Stegosauria indet.
Posteriormente, Juan Pablo Albir, de la aldea de La Almeza, (Alpuente, Valencia), nos dio a conocer la existencia de restos de dinosaurios en el lugar denominado Cerrito del Olmo, situado cerca de la mencionada aldea. En principio, se recogieron algunos cuerpos vertebrales que estaban al descubierto y se realizó una pequeña excavación obteniéndose varias vértebras cervicales características del género Dacentrurus, así como un fragmento de ísquion y varios fragmentos de costillas. El estudio de este material, en 
especial el de las vértebras cervicales completas y el del fragmento de ísquion, permitió su asignación a Dacentrurus armatus, única especie del género, característica del Jurásico Superior de Francia, Inglaterra y Portugal (Galton 1985,1991).

La última excavación efectuada en la localidad de Losilla permitió recoger nuevo material asignable al mismo taxón: vértebras cervicales, dorsales y caudales, fragmentarias en su mayoría, entre las que destaca una dorsal casi completa. El estudio de todo el material mencionado hasta ahora puede hallarse en Casanovas et al. (1995a; 1995 b y 1995c).

En este trabajo presentamos también el estudio de un fémur completo hallado posteriormente por el ya mencionado Juan Pablo Albir. Todo el material de estegosaurios hallado hasta la fecha en la comarca de Los Serranos está depositado provisionalmente en el Institut "M. Crusafont" de Sabadell, -en espera de su destino definitivo en Valencia- excepto el mencionado fémur, que pertenece a la colección particular de Juan Pablo Albir.

En síntesis, el material de estegosaurio que poseemos es el siguiente:

A) Localidad de Losilla

Lo-1. Espina caudal derecha.

Lo I-1.Fragmento de vértebra cervical posterior.

Lo I-2.Fragmento de vértebra dorsal.

Lo I-3.Vértebra dorsal casi completa.

Lo I-4.Fragmento de cuerpo de vértebra caudal.

Lo I-5.Fragmento de cuerpo de vértebra caudal.

Lo I-6.Fragmento de cuerpo de vértebra caudal.

Lo I-7.Cuerpo de vértebra dorsal.

Lo I-8.Cuerpo de vértebra dorsal.

B) Localidad de Cerrito del Olmo

CO-1. Vértebra cervical.

$\mathrm{CO}-2$. Vértebra cervical.

CO-3. Cuerpo de vértebra cervical.

CO-4. Cuerpo de vértebra dorsal.

CO-5. Cuerpo de vértebra cervical.

CO-6. Cuerpo de vértebra caudal.

CO-7. Cuerpo de vértebra dorsal.

CO-8. Cuerpo de vértebra caudal.

CO-9. Fragmento de cuerpo de vértebra cervical.

CO-10.Fragmento de cuerpo de vértebra caudal.

$\mathrm{CO}-11$. Costilla dorsal anterior.

CO-12.Costilla dorsal más posterior.

CO-13.Fragmento proximal de costilla dorsal.

CO-14.Fragmento distal de costilla.

CO-15.Fragmento distal de costilla.

CO-16.Fragmento de ísquion derecho.

CO-17.Fragmento de cuerpo vertebral.

CO-18.Fragmento de cuerpo vertebral.

CO-19.Fragmento de hueso indeterminado.

CO-20.Fragmento de hueso indeterminado.

CO-21.Fragmento de hueso indeterminado.

CO-22.Fragmento de hueso indeterminado.

$\mathrm{CO}-23$.Fragmento de cuerpo vertebral caudal.

CO-24.Fragmento de cuerpo vertebral cervical.

CO-25.Tres fragmentos de costilla.

CO II-1.Fémur derecho.

\section{GEOLOGÍA: LOS MATERIALES CON "FACIES WEALD" DE LA CUBETA DE ARAS DE ALPUENTE}

Los yacimientos con restos de dinosaurios de Losilla, La Cañada, El Losar, Los Rochones, El Castellar, Cerrito del Olmo y La Ventura están situados en el sector septentrional de la denominada "Cubeta de Aras de Alpuente", comprendido entre las poblaciones de Aras de Alpuente, Losilla de Aras, El Collado, La Almeza y La Cuevarruz (provincia de Valencia). En el entorno de estas localidades afloran materiales detríticos continentales, atribuídos a la facies Weald por González-Lodeiro et al. (1975), y asignados al intervalo de tiempo HauterivienseBarremiense por González- Lodeiro et al. (1975), Mas et al. (1982a) y Vilas et al. (1982).

Litológicamente, estos depósitos están formados por arcillas, margas y calizas, totalizando un espesor máximo de $545 \mathrm{~m}$ en el sector central de la cubeta de Aras de Alpuente (Figs. 1 y 2). Se hallan dispuestos sobre calizas

\section{Fm. Calizas de Aras de Alpuente}

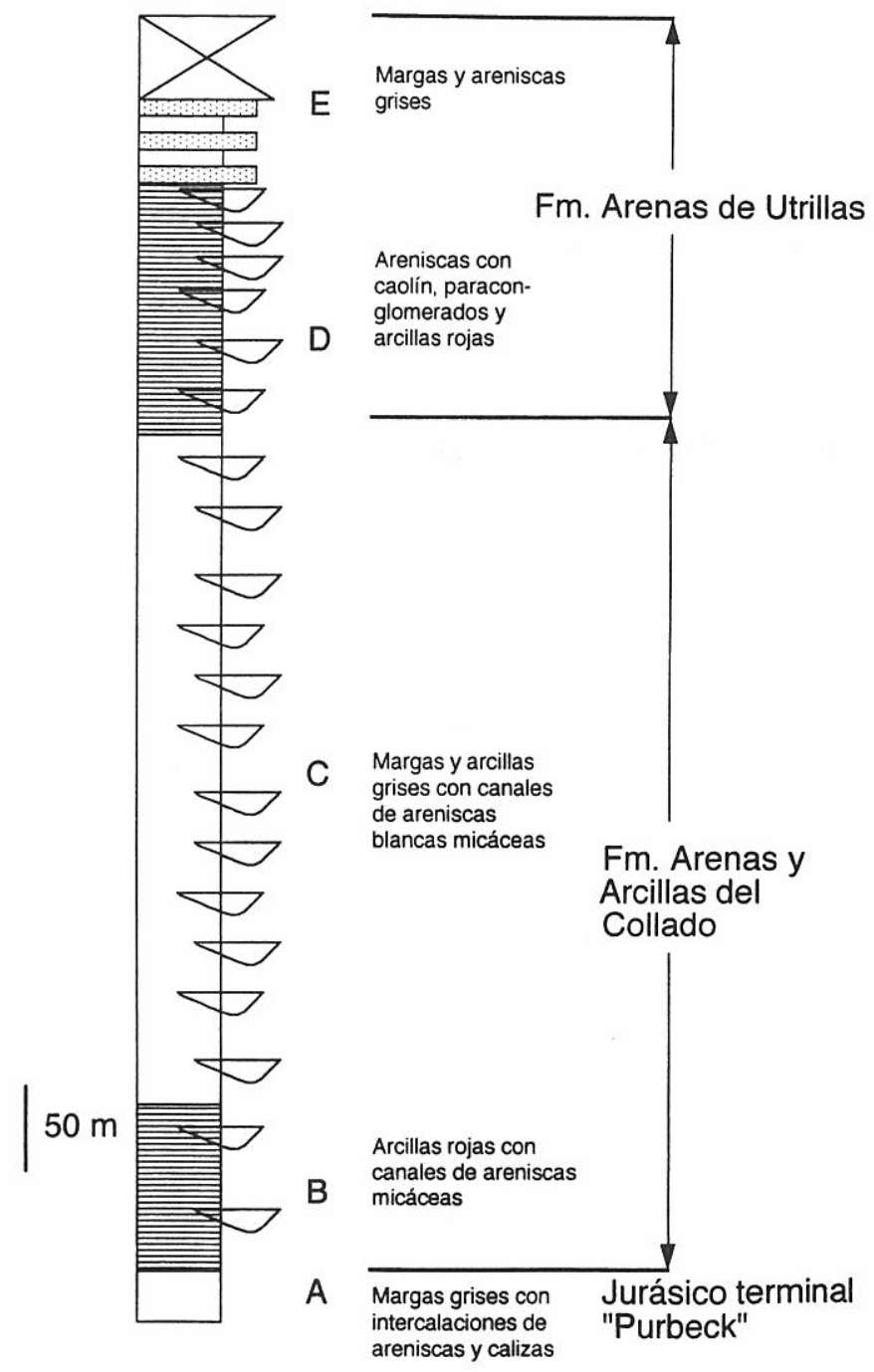

Figura 1. Columna estratigráfica de los depósitos con facies "Weald" de "La Cubeta de Aras de Alpuente". 


$$
O-E
$$

Fm. Calizas de

Aras de Alpuente
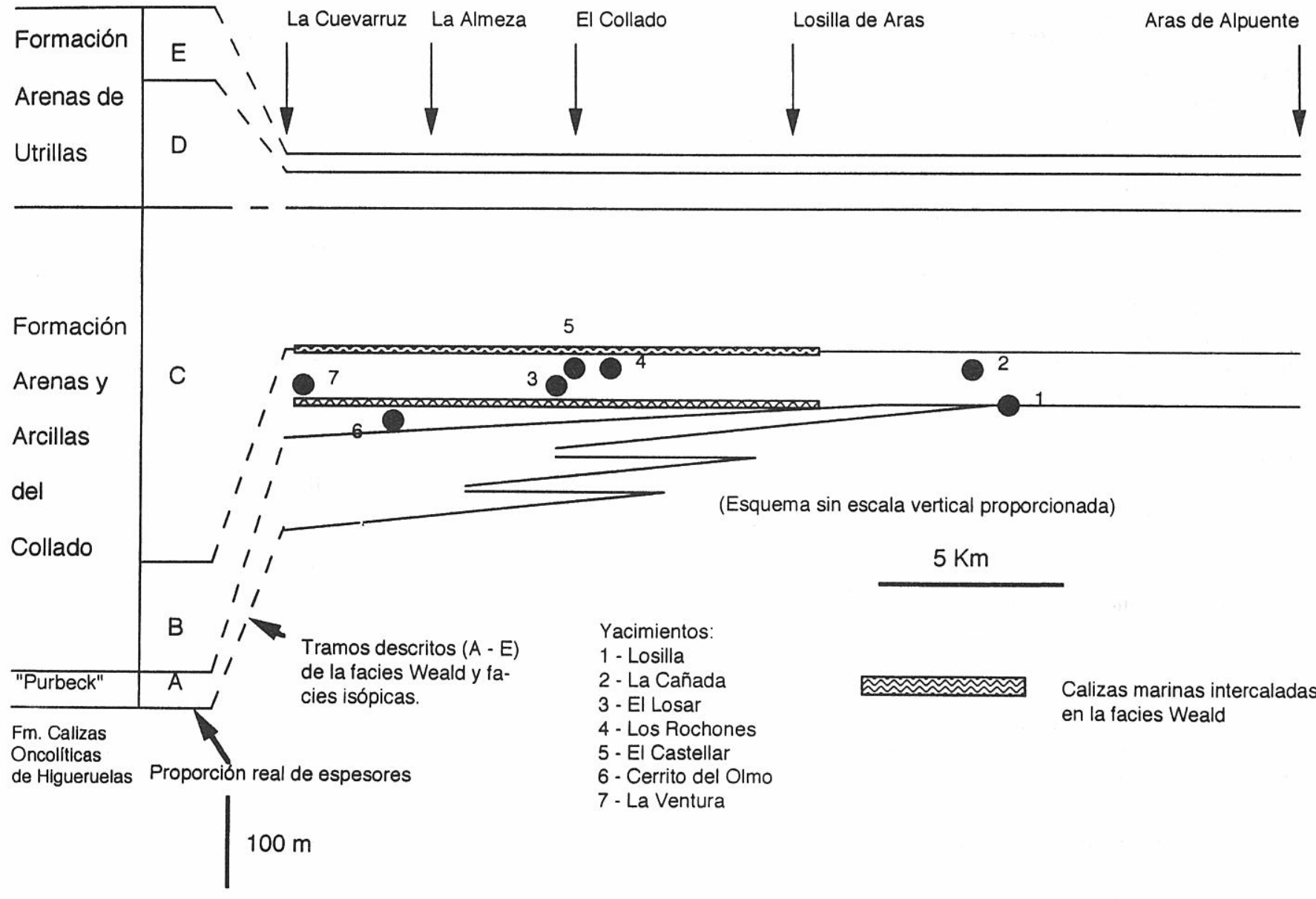

Figura 2. Correlación estratigráfica de las diversas unidades de materiales continentales del Jurásico Superior y Cretácico Inferior de la Cubeta de Aras de Alpuente, entre Aras y La Cuevarruz.

pisolíticas y oolíticas con estratificación cruzada de las facies "Purbeck" (Jurásico terminal), y sobre los materiales de la Formación Calizas Oncolíticas de Higueruelas (Mas et al., 1982b). En su techo, los depósitos con facies Weald están en contacto transicional con las arenas blancas caoliníferas, paraconglomerados y arcillas rojas de la Formación Arenas de Utrillas.

Los materiales de la facies Weald presentes en la cubeta de Aras de Alpuente, han sido asignados a la Formación Arenas y Arcillas del Collado por Vilas et al. (1982), quienes han tomado como estratotipo los afloramientos existentes entre las localidades de El Collado y La Muela. Santisteban (1995) subdivide los materiales continentales con facies tipo "Weald", comprendidos entre las formaciones Calizas Oncolíticas de Higueruelas y Calizas de Aras de Alpuente, y abarcando el intervalo de tiempo Hauteriviense (Valanginiense?)Albiense, en cinco tramos (A-E) (Figs. 1 y 2).

El tramo inferior ("A") tiene su mejor representación en el sector noroccidental de la zona (entre El Collado y La Yesa), en donde aflora con una potencia variable de 0 a 30 m. Está constituído por margas y arcillas grises, con intercalaciones de areniscas y calizas. Parte de estos materiales han sido asignados al Jurásico terminal ("Purbeck") por Mas et al. (1982b) en la localidad de El Collado, mientras que han sido cartografiados como facies Weald por González-Lodeiro et al. (1975).

El tramo "B" consiste en 115 metros de arcillas rojas conteniendo grandes cuerpos canaliformes de areniscas blancas micáceas, y dos intercalaciones de calizas grises con bioclastos marinos de un espesor entre 1 y 5 metros (Fig. 2).

El tercer tramo ("C") es el de mayor desarrollo, ya que su potencia alcanza los 400 metros. Está formado por margas y arcillas grises con intercalaciones de grandes cuerpos canaliformes de areniscas blancas micáceas con estratificación cruzada.

El tramo "D" tiene 150 metros de espesor. Está formado por un conjunto de grandes cuerpos canaliformes de arenas y areniscas blancas caoliníferas, y paraconglomerados canalizados, intercalados entre arcillas rojas.

El último tramo ("E") consiste en 90 metros de alternancias de arcillas y areniscas, organizadas en cinco secuencias granocrecientes y estratocrecientes. Estos materiales constituyen la transición entre los depósitos con facies continentales de la Formación Utrillas y los marinos de la Formación Calizas de Aras de Alpuente. 
Los depósitos del tramo "A" pasan lateralmente, mediante un sistema de "cuña en cuña", a la parte superior de la Formación Calizas Oncolíticas de Higueruelas. Debido a ello, niveles con facies tipo "Weald" se interdigitan, entre las localidades de El Collado y La Cuevarruz, con capas de calizas marinas jurásicas. Por esta razón, es posible asignar esta unidad al Jurásico terminal ("Purbeck"). Los tramos "B" y "C" son los que más claramente presentan los rasgos de la facies "Weald". Ambos se caracterizan por la presencia de grandes paleocanales de areniscas blancas intercaladas entre arcillas. Estos paleocanales poseen dimensiones variables, con un máximo de 300 metros en sección transversal, perpendicular al eje del mismo, y 40 metros de potencia. Mas et al. (1982b) han realizado una descripción detallada de uno de estos paleocanales, presente en la localidad de El Collado, que interpretan como un depósito de barra de meandro de un sistena fluvial, asociado a una llanura deltaica. Las diferencias entre los tramos " $B$ " y " $C$ " estriban en la mayor abundancia de pelitas lacustres entre los depósitos arcillosos de llanura de inundación en los materiales del tramo " $\mathrm{C}$ ".

Los tramos "E" y "D", formados por facies isópicas a la "Weald", pueden ser integrados en la Formación Arenas de Utrillas basado en un criterio de edad.

Todos los yacimientos con restos de dinosaurios estegosaurios descritos hasta la actualidad en esta zona (Casanovas-Cladellas et al., 1995a, b y c) se hallan emplazados entre materiales pertenecientes a la parte basal del tramo "B" (Fig. 2). Estos materiales contienen, en facies continentales, el límite Jurásico-Cretácico, por lo que no se puede descartar que algunos depósitos con restos de dinosaurios de las localidades estratigráficamente inferiores sean de edad Jurásico Superior.

Otros yacimientos con restos de dinosaurios están situados en una posición estratigráfica más alta dentro del tramo "B" (Fig. 2).

\section{PALEONTOLOGÍA}

Estudiado la mayor parte del material de estegosaurios hallado hasta ahora, en las publicaciones ya mencionadas en la introducción, nos limitaremos aquí al estudio del material inédito, un fémur procedente de Cerrito del Olmo II.

Se trata de una pieza columnar, esbelta y de una longitud de $92 \mathrm{~cm}$ (Lám. I, figs. 3-6). Su región proximal es aplanada anteriormente y suavemente cóncava posteriormente. La cabeza del fémur no destaca particularmente ya que está separada del gran trocánter por una débil concavidad. El trocánter menor está unido al anterior y apenas puede distinguirse. Todo el extremo superior de la región proximal es fuertemente rugoso y forma una banda también rugosa que desciende por la cara lateral hasta una distancia de 203 $\mathrm{mm}$ de la epífisis proximal. Esta banda es de forma triangular, más ancha y de más relieve en el extremo proximal, mientras que adelgaza y se estrecha hacia abajo. La cara anterior es también rugosa y la posterior lisa.

La diáfisis es estrecha, con una sección elipsoidal de 125 $\mathrm{mm}$ de diámetro transverso mínimo, por $74 \mathrm{~mm}$ de diámetro anteroposterior. No se distingue apenas el cuarto trocánter, salvo por la existencia de un pequeño saliente en la zona media de la diáfisis. Las caras medial y lateral de la pieza son divergentes, de modo que las caras anterior y posterior se estrechan en el centro de la diáfisis, ensanchándose en las zonas proximal y distal. En la cara anterior de la diáfisis, existe un cordón vertical que alcanza $600 \mathrm{~mm}$ de longitud. El hueso, dividido en dos partes en la zona media de la diáfisis, muestra una elipse cuyo exterior está formado por una lámina de hueso compacto que encierra una cavidad medular relativamente grande. Este carácter, según Galton y Boiné (1980, pág. 39), confirma la pertenencia de este espécimen a los estegosaurios y no a los saurópodos, cuya cavidad medular es muy reducida debido al grosor del hueso compacto que ocupa la mayor parte de la diáfisis.

Los cóndilos de la región distal están poco marcados anteriormente y bien señalados posteriormente. El diámetro antero-posterior del cóndilo interno es de $175 \mathrm{~mm}$ y el del externo, de $144 \mathrm{~mm}$. Este último presenta una protuberancia saliente en su cara posterior. Ambos cóndilos tienen terminaciones rugosas. El canal intercondilar es ancho pero poco profundo.

\section{DISCUSIÓN Y CONCLUSIONES}

El fémur CO-II-1 pertenece claramente a un Dinosaurio estegosaurio, como lo demuestra la forma columnar de su diáfisis, el escaso desarrollo de su cuarto trocánter, su bien definida cavidad medular y la forma de la región distal con canal intercondilar poco profundo posteriormente y menos profundo aún anteriormente (Galton, 1983).

El fémur de Cerrito del Olmo es morfológicamente comparable con el fémur figurado por Zbyszewski (1946) y revisado por Lapparent y Zbyszweski (1957), del yacimiento de Baleal (Peniche, Portugal), asignado a Omosaurus armatus Owen, 1875, que Galton, en 1985, atribuye a Dacentrurus armatus. No obstante, presenta algunas diferencias que pueden sintetizarse del modo siguiente:

a) Menor desarrollo de la cabeza del fémur del ejemplar de Cerrito del Olmo.

b) Menor longitud total en relación con el diámetro transverso de la epífisis proximal.

c) En el fémur de Portugal, existen dos cordones paralelos, muy desarrollados, en la cara posterior, que no se observan en CO-II-1. Sin embargo, un cordón longitudinal recorre la mayor parte de la cara anterior de ambos fémures.

Lámina I. Dacentrurus armatus (Owen). La escala representa $5 \mathrm{~cm}$.

1-2 CO-2. Vértebra cervical. Norma cefálica y lateral.
3-6 CO-II-1. Fémur derecho. Norma lateral, anterior, posterior, superior e inferior. 
Lámina I
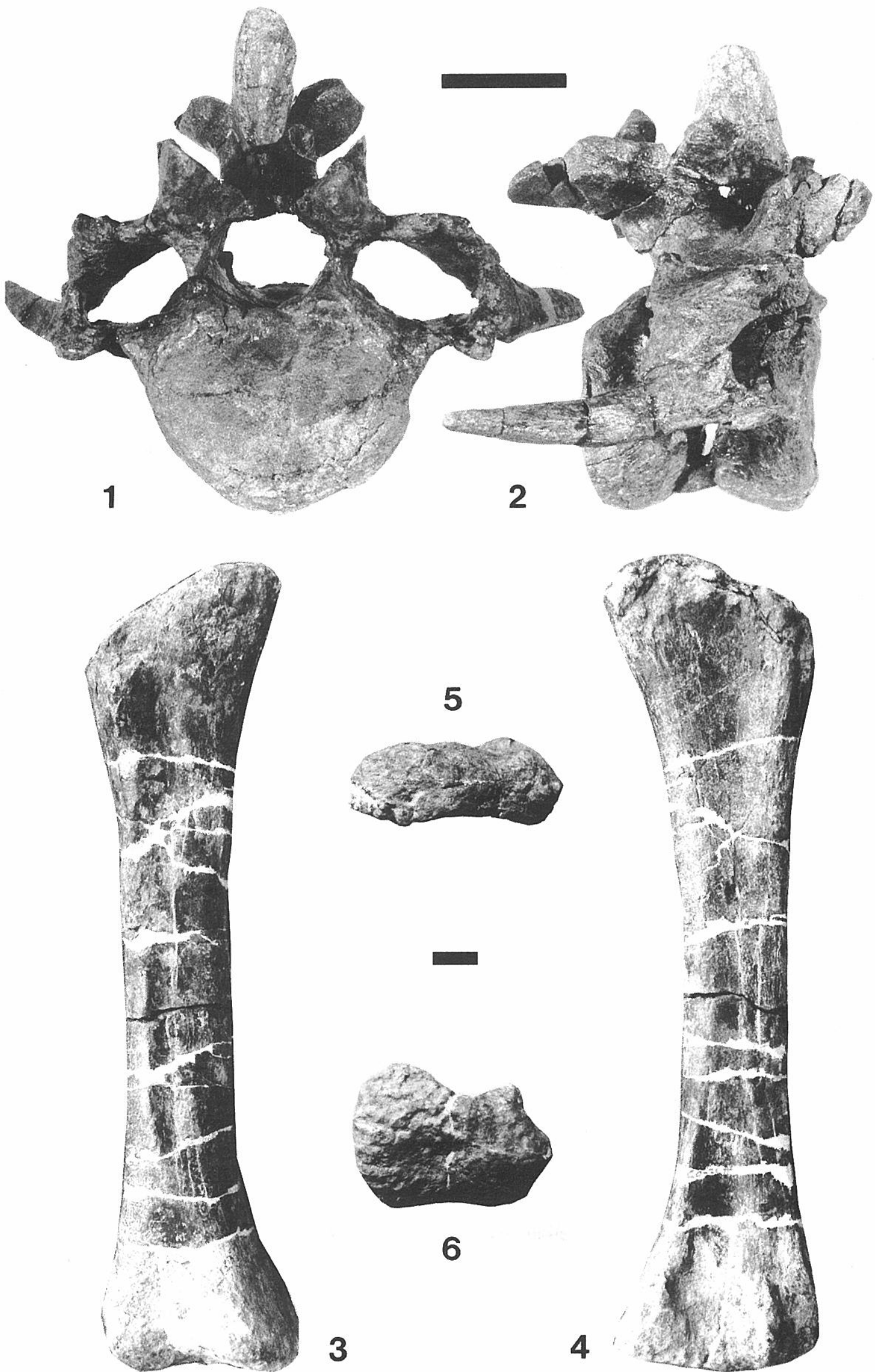

in
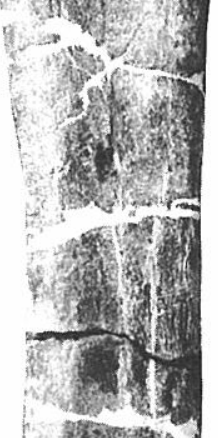

1)
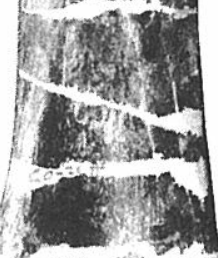

6

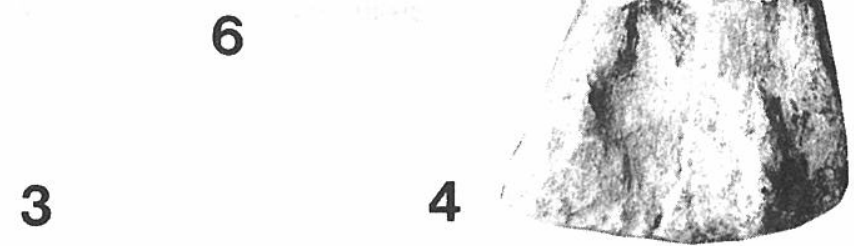

4

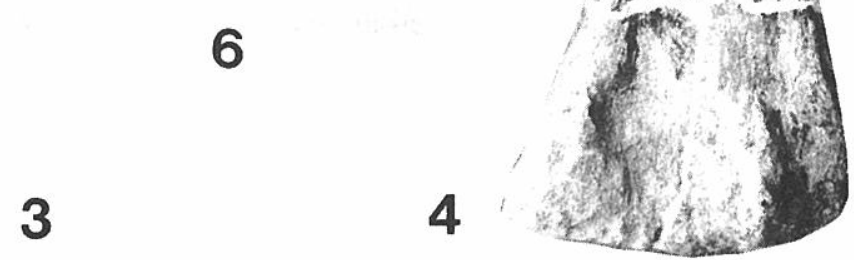

Revista Española de Paleontología, nº extr. Homenaje al Prof. J. Truyols, 1999. 
Galton, en 1982 (pág. 145) y 1991 (pág. 316) estableció las diferencias morfológicas que presentan los fémures de estegosaurios adultos y subadultos, de este modo:

a) La cabeza es mucho más esférica y está más claramente definida en los adultos que en los subadultos.

b) El trocánter menor es un proceso esbelto en forma de dedo en los subadultos, mientras que en los adultos está fusionado con el gran trocánter.

c) Proximalmente, la superficie lateral es lisa en los subadultos mientras que, en los adultos, está cubierta por protuberancias óseas.

d) La relación entre el diámetro transverso de la diáfisis y el de las regiones proximal y distal es menor en los subadultos que en los adultos, por lo que el fémur resulta más esbelto en los primeros.

e) Presencia de ligamentos osificados longitudi-nales en la diáfisis de los adultos pero no en los subadultos.

f) Prácticamente no hay señal de cuarto trocánter en la mayoría de los géneros de estegosaurios subadultos, mientras que en los adultos existe un cuarto trocánter, aunque poco desarrollado.

Galton, en 1985 (pág. 234), hace hincapié de nuevo en estos caracteres estudiando su variación en Dacentrurus, Kentrosaurus, Stegosaurus y Lexovisaurus, observando que, en Stegosaurus, el cuarto trocánter falta incluso en los individuos adultos, y, en su lugar, hay rugosidades en el área apropiada.

Todas estas particularidades morfológicas se manifiestan en nuestro ejemplar del siguiente modo:

a) La cabeza del fémur es poco esférica y está poco definida.

b) El trocánter menor está relativamente fusionado con el gran trocánter.

c) La superficie lateral proximal presenta protuberancias óseas, pero éstas no son muy abundantes.

d) La relación longitud/diámetro transverso de la diáfisis es menor en nuestro ejemplar que en el fémur de Portugal.

e) Existe un único cordón longitudinal en la cara anterior de la diáfisis.

f) El cuarto trocánter, situado en la zona media de la diáfisis, está muy poco marcado.

Galton y Boiné (1980, pág. 37) hablan del escaso desarrollo del cuarto trocánter de todos los fémures de Dacentrurus descritos de Portugal. Los autores indican que ello es debido probablemente a la diferencia de talla, ya que al compararlos con otros ejemplares de talla mayor-BMNH 46013, holotipo del género, o con el fémur de La Brière, Francia- éstos presentaban un cuarto trocánter. Los mismos autores creen que el fenómeno se produce también en Kentrosaurus y Lexovisaurus, pero piensan que la talla mínima de los fémures con un cuarto trocánter prominente es diferente en cada género.

Por otra parte, Hennig (1925) hace notar que existe toda una serie de variaciones individuales, por lo menos en Kentrosaurus, ya que fémures de mayor longitud presentaban rugosidades insignificantes, mientras que otros más cortos tenían un trocánter saliente.

A la vista de todo lo expuesto, y basándose en la presencia de material diagnóstico en la misma área, el fémur de Cerrito del Olmo puede ser asignado a Dacentrurus armatus (Owen). Se trataría de una pieza perteneciente a un individuo adulto, a pesar de que la cabeza del fémur está poco definida y que el cuarto trocánter está apenas señalizado. Los demás criterios concuerdan con la definición de Galton, $(1982 ; 1985)$ para diferenciar los individuos adultos de los subadultos.

En conclusión, el material de estegosaurios de la comarca de Los Serranos estaría formado por vértebras cervicales (Lám. I, figs. 1 y 2), dorsales y caudales, costillas, un fragmento de ísquion y un fémur. Este último se describe en este trabajo por primera vez.

Todo este material se atribuye, en conjunto, a Dacentrurus armatus (Owen, 1875). En trabajos anteriores, las localidades que proporcionaron estos materiales han sido consideradas como de edad Cretácico Inferior (facies Weald). Sin embargo, no se descarta que el Dacentrurus armatus de Valencia proceda del Jurásico terminal (facies Purbeck). Esta posibilidad está más en concordancia con los datos de la distribución de Dacentrurus en Europa.

\section{AGRADECIMIENTOS}

Nuestro agradecimiento a Juan Pablo Albir, de la aldea de La Almeza, por darnos a conocer el yacimiento de Cerrito del Olmo, así como por prestarnos el fémur para su estudio. A los restauradores del Institut de Paleontologia "M. Crusafont", Isabel Pelejero, Eulàlia Soler y Angel Galobart por su trabajo de preparación del material, así como por las fotografías realizadas por este último.Finalmente, agradecemos a nuestro oficial de campo, Antonio Adell y a los restantes trabajadores de campo la ayuda prestada en los trabajos de extracción de material durante las excavacionas llevadas a cabo en la Comarca de Los Serranos.

Este trabajo es una contribución al Proyecto PB96-0102.

\section{BIBLIOGRAFÍA}

Casanovas-Cladellas, M.L., Santafé-Llopis, J.V. y PeredaSuberbiola, X.1995a. Nuevo material de estegosaurios en el Cretácico Inferior de Valencia. Paleontologia $i$ Evolució, 28-29, 269-274.

Casanovas-Cladellas, M.L., Santafé-Llopis, J.V., PeredaSuberbiola, X. y Santisteban-Bové, C. 1995b. Presencia,por primera vez en España, de dinosaurios estegosaurios. (Cretácico Inferior de Aldea de Losilla, Valencia). Revista Española de Paleontología, 10, 8389.

Casanovas-Cladellas, M.L., Santafé-Llopis, J.V. y Santisteban-Bové, C. 1995c. Dacentrurus armatus (Stegosauria, Dinosauria) del Cretácico Inferior de los Serranos (Valencia, España). Revista Española de Paleontología, 10, 273-283. 
Galton, P.M. 1982. The postcranial Anatomy of Stegosaurian Dinosaur Kentrosaurus from the Upper Jurassic of Tanzania, East Africa. Geologica et Paleontologica, 15, 139-160.

Galton, P.M. 1983. A juvenile Stegosaurian Dinosaur, Omosaurus phillipsi Seeley from the Oxfordian (Upper Jurassic) of England. Geobios, 16, 95-101.

Galton, P.M. 1985. British plated Dinosaurs (Ornithischia, Stegosauridae). Journal of Vertebrate Paleontology, 5, 211-254.

Galton, P.M. 1991. Postcranial remains of stegosaurian dinosaur Dacentrurus from Upper Jurassic of France and Portugal. Geologica et Palaeontologica, 25, 299-327.

Galton, P.M. and Boiné G. 1980. A stegosaurian Dinosaur femur from the Kimmeridgian beds (Upper Jurassic) of the Cap de la Hève, Normandy. Bulletin trimestral Societé Géologique de Normandie et Amis Muséum du Havre, 67 (4), 31-38.

González Lodeiro, F., Iglesias Ponce de León, M.,Rubio Novas, J., García Salinas, F., Abril Hurtado, J. y Ortí Cabo, F. 1975. Hoja geológica y Memoria explicativa del Mapa geológico de España, E. 1:50.000. Hoja n638, 27-25. Alpuente. Instituto Geológico y Minero de España, Madrid, 1-34.

Hennig, E. 1925. Kentrosaurus aethiopicus. Die Stegosaurier-Funde Tendaguru, Deutsch-Ostafrika.
Paleontographica 7, 101-253.

Lapparent, A.F. et Zbyszewski, G. 1957. Les Dinosauriens de Portugal. Services Géologiques du Portugal, 2, (Nouv. Série), 9-63.

Mas, J. R., Alonso, A. y Meléndez, N. 1982a. El Cretácico basal "Weald" de la Cordillera Ibérica suroccidental (NW de la provincia de Valencia y E de la de Cuenca). Cuadernos de Geología Ibérica, 8, 309-335.

Mas, J.R., Alonso, A. y Meléndez, N. 1982b. El Cretácico basal "Weald" en la transversal Valencia-Cuenca. In: II Coloquio de Estratigrafía de España. Excursión postColoquio. In: El Cretácico inferior de la Cordillera Ibérica suroccidental. Grupo Español del Mesozoico, Albacete. Editorial de la Universidad Complutense de Madrid, 29-126.

Santisteban, C. 1995. Los depósitos continentales con facies Weald de la cubeta de Aras de Alpuente, Comarca de Los Serranos, Valencia. Geogaceta, 17, 16-18.

Vilas, L., Mas, R., García, A., Arias, C., Alonso, A., Meléndez, N. y Rincón R. 1982. Capítulo 8. Ibérica Suroccidental. In: El Cretácico de España. Editorial de la Universidad Complutense de Madrid, 457-509.

Zbyszewsky, G. 1946. Les ossements d'Omosaurus découverts près de Baleal (Peniche). Comunicaçoes dos Serviços Geológicos de Portugal, 27, 3-12. 